\title{
Gorlin syndrome - an incidental radiographic detection
}

\section{Shishir Ram Shetty, MDS}

Department of Oral Medicine and Radiology, AB Shetty Memorial Institute of Dental Sciences, Mangalore, India

\section{K M Veena, MDS \\ Laxmikanth Chatra, MDS \\ Prashanth Shenai, MDS \\ Prasanna Kumar Rao, MDS}

Department of Oral Medicine and Radiology, Yenepoya Dental College, Deralakatte, Mangalore

\section{Jagadish Chandra, MDS}

Department of Oral and Maxillofacial Surgery, Yenepoya Dental College

\section{Introduction}

Gorlin-Goltz syndrome (also known as nevoid basal cell carcinoma syndrome) was first reported in 1894, but described by Gorlin and Goltz in 1960 as a distinct entity consisting of ectodermal and mesodermal abnormalities. ${ }^{1}$ It is an hereditary autosomal dominant disease with a prevalence estimated in various studies to be between 1/57 000 and $1 / 256000$, and a male:female ratio of $1: 1 .^{2}$ We describe in brief the important radiological features of an accidentally detected case of Gorlin syndrome in the form of a pictorial interlude.

\section{Case report}

A 28-year-old abnormally tall man with forwardly displaced teeth presented to us. As part of pre-treatment evaluation, a panoramic radiograph was made. Well-defined cyst-like radiolucent areas which were associated with impacted third molars were observed in all quadrants of the jaw (Fig. 1). Evoking suspicion of a syndrome, a thorough general examination was performed. The patient had long, slender, fused fingers and toes, and multiple naevi measuring 2 - 3 $\mathrm{mm}$ over the skin. A chest radiograph revealed T1 splitting fusion and forking of the 3rd and 4th right ribs (Figs 2 and 3). A hand-wrist radiograph showed localised gigantism of the $2 \mathrm{nd}$ and 3rd digits with flexion deformity at the proximal metacarpo-phalangeal joints and soft

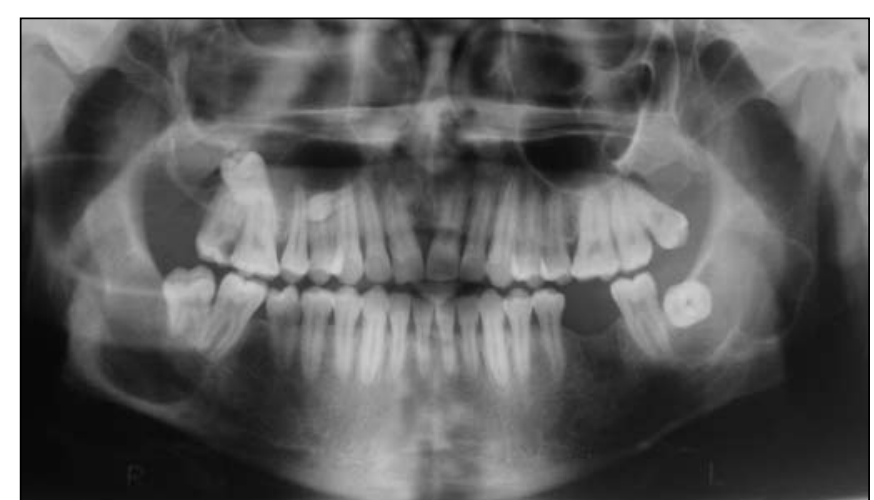

Fig. 1. Panoramic view showing cyst-like radiolucencies in all quadrants of the jaws. Note also impacted 3rd molars associated with the radiolucencies.

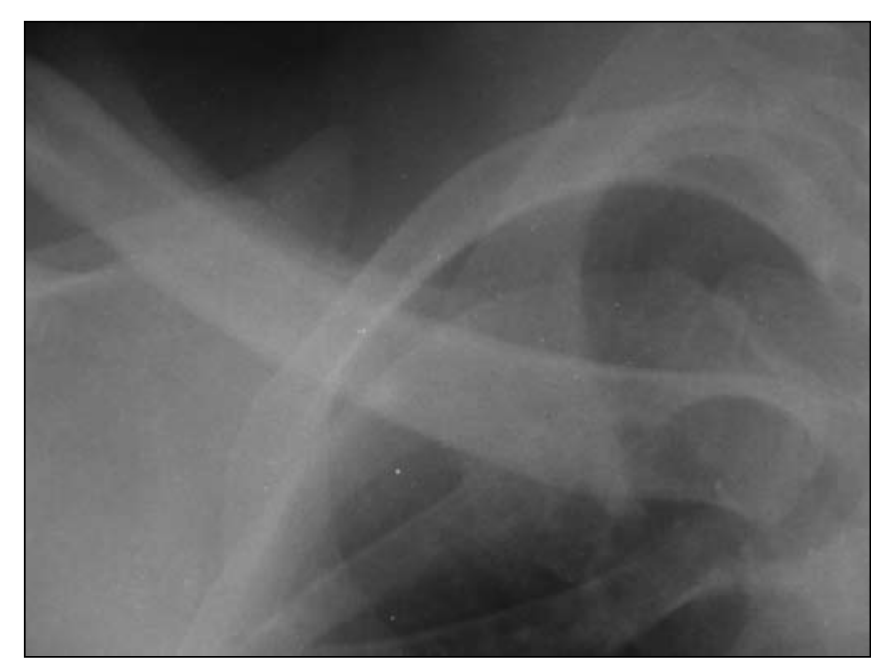

Fig. 2. Cropped section of chest radiograph showing abnormal rib splitting.

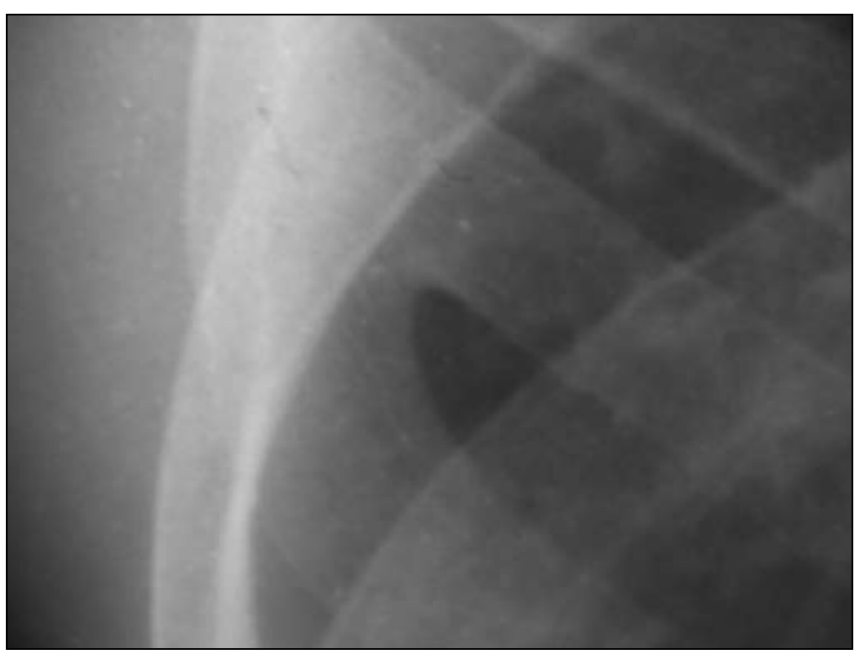

Fig. 3. Cropped section of chest radiograph showing forking of the 4th thoracic rib. 


\section{PICTORIAL INTERLUDE}

tissue syndactyly of the 4th and 5th fingers, with fusion of the terminal phalanges. Alignment deformity of the distal interphalangeal joints of the 2nd and 3rd fingers was also observed (Fig. 4).

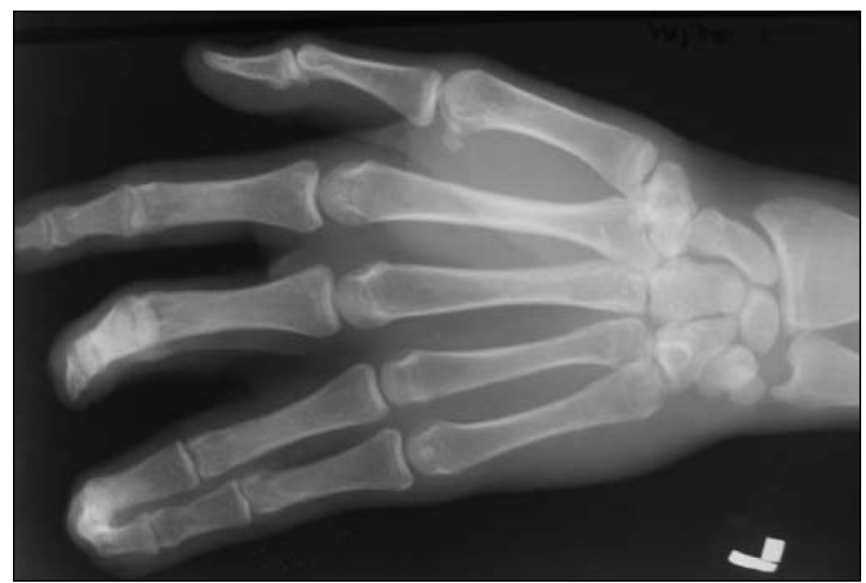

Fig. 4. Hand-wrist radiograph showing bony fusion of the terminal 4 th and 5th terminal phalanges.

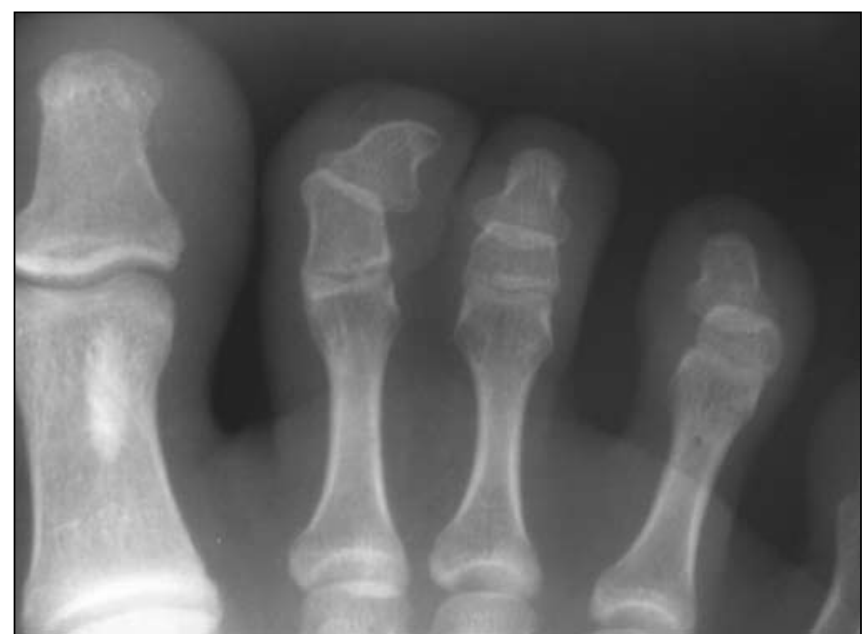

Fig. 5. Radiograph showing soft-tissue fusion of 2 nd and $3 r d$ toes. Note too bone island on the big toe.
Radiography of the feet revealed soft-tissue syndactyly of the 2nd and 3rd toes bilaterally and a bone island on the proximal phalanx of the big toe (Fig. 5). Based on these clinical and radiological findings, a diagnosis of Gorlin syndrome was made. No evidence of calcification of falx cerebri was detected during the CT scan. Surgical excisions of the cysts were performed under general anaesthesia. Histopathological examination of the excised specimens revealed features of odontogenic keratocyst. The patient did not agree to surgical correction of the finger and toe deformities. Unfortunately, he could be followed up for only 6 months, during which time no relapse was observed.

\section{Discussion}

Gorlin syndrome (also known as Gorlin-Goltz syndrome) is a condition characterised by multiple odontogenic keratocysts, basal cell carcinoma, congenital skeletal abnormalities, cleft lip and/or palate, and eye anomalies. Various low-frequency neoplasms, such as meningiomas and ovarian and cardiac fibromas, have been also reported. ${ }^{3}$ Our patient presented with features of multiple keratocysts in all quadrants of the jaw, skin and skeletal abnormalities, so fulfilling 2 major and 1 minor criteria. ${ }^{4}$ Keratocysts are present in $90 \%$ of cases of Gorlin syndrome, and are most commonly seen in the molar-ramus areas. ${ }^{5}$ Similar areas were involved in our case. Rib and finger abnormalities are noticed in $30-40 \%$ of cases. $^{6}$ Similar findings were detected in our case. Our case highlights the key radiological features in the form of a pictorial interlude.

1. Gorlin RJ, Goltz RW. Multiple nevoid basal-cell epithelioma, jaw cysts and bifid rib: a syndrome. N Engl J Med 1960;262:908-912

2. Lo Muzio L. Nevoid basal cell carcinoma syndrome (Gorlin syndrome). Orphanet J Rare Dis 2008;25(3):32.

3. Evans DG, Ladusans EJ, Rimmer S, et al. Complications of the naevoid basal cell carcinoma syndrome: results of a population based study. J Med Genet 1993;30:460-464.

4. Kimonis VE, Mehta SG, Digiovanna JJ, et al. Radiological features in 82 patients with nevoid basal cell carcinoma (NBCC or Gorlin) syndrome. Genet Med 2004;6(6):495-502.

5. Muzio L, Nocini P, Bucci P, et al. Early diagnosis of nevoid basal cell carcinoma. J Am Dent Assoc 1999;130(5):669-674.

6. Muzio L, Nocini P, Bucci P, et al. Nevoid basal cell carcinoma syndrome. Clinical findings in 37 Italian affected individuals. Clin Genet 1999;55(1):34-40. 